
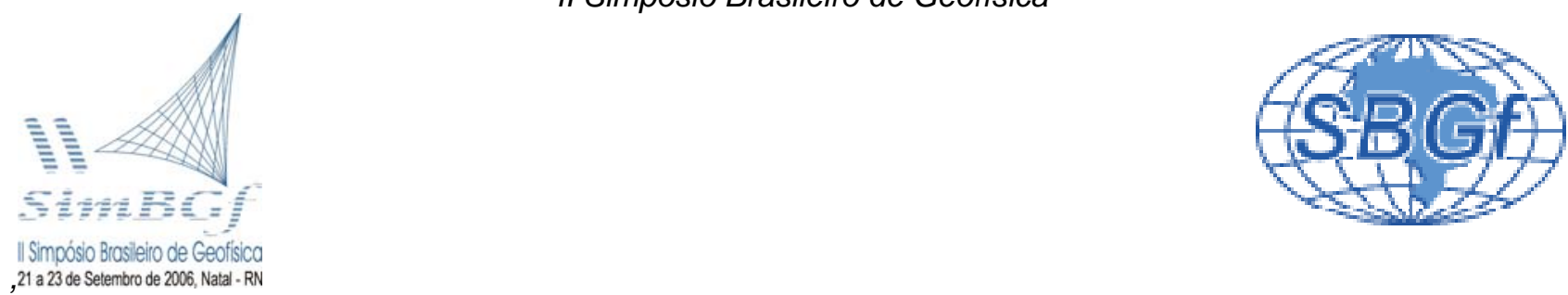

\title{
ARQUEOINTENSIDADE NO BRASIL: PRIMEIROS RESULTADOS
}

\author{
Edgard L. Catelani ${ }^{1}$, Gelvam A. Hartmann ${ }^{1}$, Ricardo I. F. Trindade ${ }^{1}$, Marisa C. Afonso ${ }^{2}$. \\ ${ }^{1}$ Departamento de Geofísica, Instituto de Astronomia, Geofísica e Ciências Atmosféricas, Universidade de São Paulo. \\ ${ }^{2}$ Museu de Arqueologia e Etnologia, Universidade de São Paulo.
}

Copyright 2006, SBGf - Sociedade Brasileira de Geofísica

Este texto foi preparado para a apresentação no II Simpósio de Geofísica da Sociedade Brasileira de Geofísica, Natal, 21-23 de setembro de 2006. Seu conteúdo foi revisado pela Comissão Tecno-científica do I I SR-SBGf mas não necessariamente representa a opinião da SBGf ou de seus associados. E proibida a reprodução total ou parcial deste material para propósitos comerciais sem prévia autorização da SBGf

\section{Resumo}

A grande escassez de dados arqueomagnéticos no hemisfério sul do planeta dificulta a determinação de modelos acurados para a evolução do campo magnético terrestre nos últimos milênios. Neste trabalho apresentamos a técnica utilizada para recuperar a intensidade do campo arqueomagnético e os primeiros resultados arqueomagnéticos no Brasil, utilizando material cerâmico histórico e pré-histórico dos acervos do MAE/USP e do MAE/UFBA.

\section{Introdução}

O campo magnético terrestre apresenta variações em direção e intensidade em escalas de tempo que vão de alguns segundos a milhões de anos. As variações ocorridas em décadas ou séculos correspondem às variações arqueomagnéticas e podem ser usadas para investigar os processos de formação do campo magnético terrestre, bem como servir de base para a datação magnética de artefatos arqueológicos.

Embora existam curvas de variação para a Europa e o oeste da Ásia, os dados advindos da América do Sul são ainda bastante escassos (menos de 1\% do banco de dados mundial). Neste sentido foi iniciado há dois anos um projeto para estudos arqueomagnéticos no Brasil, onde estão sendo investigadas as variações na intensidade do campo magnético terrestre na América do Sul nos últimos dois milênios. Para tal, estão sendo utilizados artefatos cerâmicos submetidos a aquecimento (telhas, tijolos, utensílios) com idade dentro deste intervalo. Esses fragmentos provêm de sítios arqueológicos do Sudeste e Nordeste do Brasil e pertencem aos acervos do MAE/USP e do MAE/UFBA.

$A$ intensidade do paleocampo magnético pode ser obtida a partir do método Thellier-Thellier, no qual a magnetização original da rocha $\left(M_{\mathrm{rn}}\right)$ é gradativamente substituída por uma magnetização termoremanescente artificial adquirida em campo controlado (ver adiante em maior detalhe).

Até o momento o trabalho envolveu a modificação de um forno paleomagnético, com a inclusão de um sistema para indução de magnetização com campo magnético controlado e uniforme (variação de menos de $2 \mu \mathrm{T}$ em um comprimento útil de $40 \mathrm{~cm}$ ) e a análise de um conjunto de amostras-piloto de Casas Bandeiristas de São Paulo (séc. XVII e XVIII), Casas Coloniais da Bahia (séc. XVI e XVII), e cerâmicas Kaingang do Vale do Ribeira (séc. XII a XVI).

\section{Método Thellier-Thellier}

No momento em que uma rocha magmática ou um artefato cerâmico, que possui minerais ferromagnéticos, é resfriado na presença de um campo magnético a partir de sua temperatura de bloqueio $\left(T_{B}\right)$, ele adquire uma magnetização termoremanescente (TRM). Esta TRM é proporcional ao campo magnético ambiente indutor. Portanto, conhecendo-se a constante de proporcionalidade de um dado material, pode-se determinar o campo indutor a partir do valor de seu TRM.

Com o intuito de substituir a magnetização remanescente natural (MRN) por magnetizações termoremanescentes parciais (pTRMs) aplica-se um campo conhecido $\left(\mathrm{H}_{\mathrm{lab}}\right)$ - estabelecendo assim uma razão entre a MRN e a TRM $\mathrm{TRa}_{\mathrm{lab}}$ - o método ThellierThellier utiliza o aquecimento progressivo de uma determinada amostra até o ponto de Curie e a aplicação de um $\mathrm{H}_{\mathrm{lab}}$ durante $\mathrm{o}$ resfriamento. A cada passo estabelecido essas pTRMs são adquiridas em intervalos de temperaturas, como por exemplo entre $550^{\circ} \mathrm{C}$ e $500^{\circ}$ C. Para cada intervalo os valores de pTRM são independentes dos demais passos. Deste modo, pode-se obter diversas estimativas independentes do paleocampo para cada amostra. :

$$
M R N=\alpha_{n} H_{n} \quad \text { e } \quad T R M_{l a b}=\alpha_{l a b} H_{l a b},
$$


nas quais $\boldsymbol{H}_{\boldsymbol{n}}$ é o campo indutor natural (terrestre), $\boldsymbol{H}_{\text {lab }}$ é o campo indutor gerado no laboratório e $\boldsymbol{\alpha}_{n}$ e $\boldsymbol{\alpha}_{l a b}$ são as constantes de proporcionalidade entre magnetizaao remanescente e campo indutor. Para uma mesma amostra $\boldsymbol{\alpha}_{n}=\boldsymbol{\alpha}_{l a b}$. Portanto:

\section{$H_{n}=H_{l a b}\left(M R N / T R M_{l a b}\right)$}

A estimativa do valor de paleocampo é efetuada a partir da representação da $T R M_{\text {lab }}$ adquirida contra a $M R N$ restante a cada passo (Figura 1). Neste diagrama os pontos devem se alinhar em uma reta com coeficiente angular $\boldsymbol{H}_{n} / \boldsymbol{H}_{\text {lab. }}$.

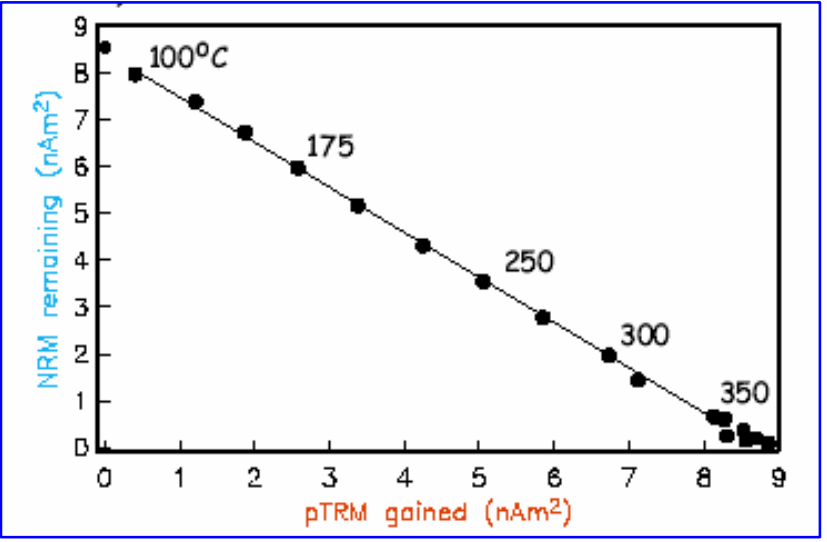

Figura 1. Diagrama de Arai, onde são representadas as remanescências artificial (pTRM) e natural (NRM) a cada passo de aquecimento. Note que os pontos alinham-se em uma reta, obedecendo à relação linear entre magnetização adquirida e campo indutor.

Neste trabalho nós utilizamos a versão de Coe (1967) e Aitken (1988) do método desenvolvido originalmente por Thellier e Thellier (1938). Neste método a primeira etapa de aquecimento corresponde à indução de uma pTRM. Em seguida efetua-se a desmagnetização térmica na mesma temperatura, obtendo-se diretamente a MRN restante com cada etapa. Dessa maneira, as duas relações são:

$$
M_{1}=M R N_{\text {restante }}+p T R M \text { e } M_{2}=M R N_{\text {restante }}
$$

Além dessas duas magnetizações, a cada dois passos de indução foram determinados pTRM checks, que são induções efetuadas a temperaturas mais baixas, para verificar a reprodutibilidade das pTRMs adquiridas e assim identificar eventuais alterações térmicas induzidas durante os aquecimentos sucessivos. Um exemplo obtido em cerâmicas históricas no sítio da Praça da Sé, Salvador, é apresentado na Figura 2.
Figura 2. Diagrama de Arai para sítio da Praça da Sé. As linhas com triângulos indicam os pTRM checks. Note a excelente concordância entre os checks e os respectivos passos de aquisição, indicando a ausência de alteração térmica. O campo calculado para este espécimen (SSI), utilizando todo o espectro de temperaturas $\left(0-520^{\circ} \mathrm{C}\right)$, foi de $26.69 \pm 1.26 \mu T$.

\section{Instrumentação}

A obtenção de dados de paleointensidade no laboratório de paleomagnetismo do IAG/USP envolveu a adaptação de todos os sistemas de medida, desde os fornos paleomagnéticos até o magnetômetro criogênico. O forno paleomagnético, com elemento aquecedor cilíndrico, foi modificado de modo a incorporar uma bobina indutora de campo, que gera um campo uniforme ao longo de $40 \mathrm{~cm}$ de seção da câmara de aquecimento. Este campo é regulado a partir de um circuito desenvolvido especificamente para este fim, conectado a uma fonte de corrente. O campo de laboratório utilizado nas medidas foi de $30 \mu \mathrm{T}$. Além da bobina indutora foram acrescentados ao forno três termopares (tipo J) para controle de temperatura ao longo de toda a câmara aquecedora, permitindo identificar eventuais gradientes térmicos durante 0 aquecimento das amostras. As amostras foram posicionadas no setor com menor gradiente térmico do forno. Em função da pequena quantidade de material disponível para as análises (fragmentos cerâmicos por vezes com menos de $0,5 \mathrm{~cm}$ de espessura), do limite da zona estável de temperatura no forno e tendo em vista a grande sensibilidade do magnetômetro criogênico, optou-se por amostras amostras cilíndricas de $1 \mathrm{~cm}^{3}$, bem menores que as amostras padrão do paleomagnetismo (de $\sim 10 \mathrm{~cm}^{3}$ ). Isso exigiu o desenvolvimento de porta-amostras especiais tanto para o forno quanto para o magnetômetro. 
Foram obtidos resultados em amostras cerâmicas históricas e pré-históricas e em amostras de rocha, onde foram induzidos campos de laboratório conhecidos de $30 \mu \mathrm{T}$ e $50 \mu \mathrm{T}$. As intensidades obtidas nas amostras com campo indutor conhecido reproduziram os valores de campo de laboratório com incerteza inferior a $5 \%$, o que permitiu validar o protocolo de medidas utilizado. Os resultados obtidos em cerâmicas históricas e pré-históricas apresentaram erros inferiores a $5 \%$ e diferenças inferiores a $10 \%$ para amostras de um mesmo sítio. Esses resultados satisfazem os critérios de qualidade estabelecidos para este tipo de medida.

Os resultados revelaram campos baixos (da ordem de $30 \%$ do campo atual) para as amostras de cerâmicas indígenas, mais antigas, campos relativamente altos para as cerâmicas do séc. XVII (cerca de 100\% mais elevado que o campo atual) e campos muito próximos do campo atual no material do séc. XVIII. As amostras dos sítios paulistas e baianos apresentam coerência temporal, permitindo traçar uma curva arqueomagnética preliminar para o intervalo entre $1100 \mathrm{e}$ 1800 AD (Figura 3). Esta curva indica um aumento significativo das intensidades entre 1100 e 1600 AD, marcado peos baixos valores de campo, entre 8 e $22 \mu \mathrm{T}$ obtido nas cerâmicas indígenas, seguidos pelos valores elevados obtidos nas cerâmicas históricas de Bahia, com valores de até $65 \mu \mathrm{T}$. A partir de então, os valores decrescem até valores em torno de $35 \mu \mathrm{T}$ em $1800 \mathrm{AD}$, obtidos nas casas bandeiristas de São Paulo, acompanhando a tendência de declínio do dipolo terrestre identificada nos laboratórios geomagnéticos a partir do século XIX. Isto resulta numa evolução significativamente diferente daquela descrita para 0 hemisfério norte, que tem uma queda suave até $1800 \mathrm{AD}$ e então uma queda acentuada até os dias atuais (Gubbins et al., 2006).

Por fim, esses dados podem ser comparados com modelos desenvolvidos a partir da interpolação de dados de navegadores desde o século XVI (Barraclough, 1974). Nota-se que a evolução traçada pelos dados arqueomagnéticos mimetiza aquela indicada pelos modelos, com valores máximos de intensidade entre 1600 e $1700 \mathrm{AD}$ e uma queda até os dias atuais.

\section{Agradecimentos}

Os autores agradecem o apoio financeiro da FAPESP (Processos 05/50257-1, 05/57782-4), ao Programa USPCOFECUB (Arqueomagnetismo no Brasil) e ao CNPq pela bolsa de Produtividade em Pesquisa (RIFT).

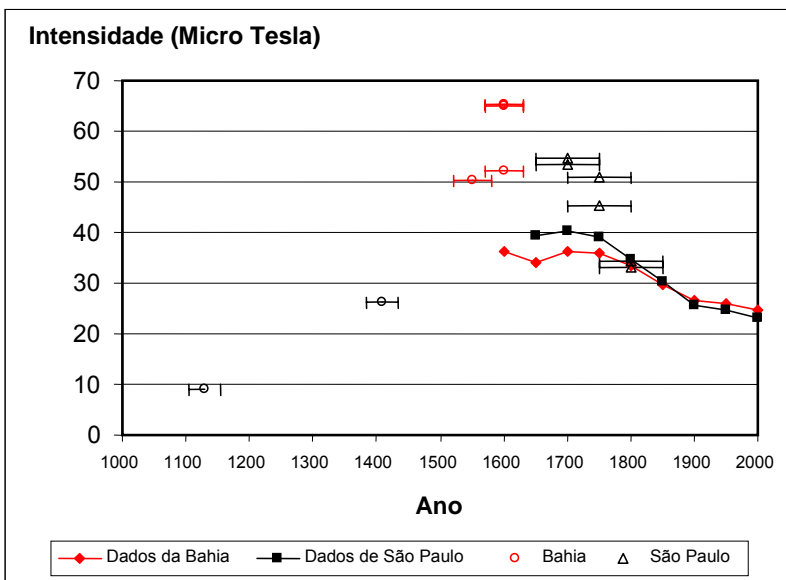

Figura 2. Evolução da intensidade com o tempo para São Paulo (azul) e Bahia (vermelho). Os pontos com barras de erro são as determinações obtidas neste trabalho. Os pontos ligados por linhas representam os valores médios de campo em São Paulo e na Bahia até $1600 A D$ obtidos com dados de navegadores e interpolados a cada 50 anos. As estimativas a partir de $1900 A D$ foram obtidas a partir de dados de observatórios. Note a evolução semelhante obtida com os dados de arqueointensidade e com as curvas, muito embora os dados de arqueointensidade apresentem valores sistematicamente mais elevados que os modelos para idades entre 1600 e 1700 AD.

\section{Referências}

Aitken, M., Allsop, A., Bussel, G., Winter, M.B. 1988. Determination of the intensity of the Earh's magnetic field during archaeological times: reliability of the Thellier technique. Rev. Geophys., 26, 3-12.

Barraclough, D.R. 1974. Spherical Harmonic Analyses of the Geomagnetic Field for Eight Epochs between 1600 and 1910. Geophys. J. R. Astron. Soc., 36, 497-513.

Coe, R.S. 1967. The determination of paleo-intensities of the Earth's magnetic field with emphasis on mechanisms which could cause non-ideal behavior in Thelier's method. Journal Geomagn. Geoeletric., 19, 157-179.

Gubbins, D., Jones, A.L., Finlay, C.C. 2006. Fall in Earth's magnetic field is erratic. Science 312: 900-902.

Thellier, E. e Thellier, O. 1938. Sur l'intensité du champ magnetiqué terrestre dans le passé historique et géologique. Ann. Geophys., 15, 285-376, 1959. 